\title{
Caracterización y análisis comparativo de biodiesel a base de higuerilla B10, respecto de diésel comercial de alta calidad
}

\section{Characterization and comparative analysis of castor oil biodiesel B10 relative to high quality commercial diesel}

\author{
Carlos Nolasco Mafla Yepez \\ Ignacio Bayardo Benavides Cevallos \\ Erik Paul Hernández Rueda \\ Fernado Renato Ramirez Paredes \\ Universidad Técnica del Norte, Ecuador
}

Autor para correspondencia: utcnmafla@utn.edu.ec, ibbenavides@utn.edu.ec, ephernandez@utn.edu.ec, frramirez@utn.edu.ec

Fecha de recepción: 20 de Enero de 2018 - Fecha de aceptación: 25 de julio de 2018

Resumen: En este artículo se presenta la caracterización fisicoquímica de diésel comercial (Premium), y de biodiesel a base de aceite de higuerilla, en proporción B10. Se ha realizado un análisis comparativo en cuanto a las características obtenidas para ambos tipos de combustible y se muestran los resultados alcanzados. Las propiedades fisicoquímicas tratadas son: Punto de inflamación, viscosidad cinemática, sedimentos básicos y agua (BSW), corrosión a la lámina de cobre, azufre e índice de cetano calculados. Las propiedades del biodiesel están influenciadas por las características físicas de la composición de ácidos grasos, tales como el grado de insaturación, el porcentaje de ácidos grasos saturados, ácidos grasos mono-insaturados, y el ácido graso poliinsaturado. Las propiedades de los combustibles son los factores clave para determinar la idoneidad de cualquier combustible alternativo. Los resultados muestran que las características del biodiesel B10 están dentro de los rangos estipulados por normativa internacional, además de reducir significativamente emisiones contaminantes. La caracterización de los combustibles ha sido realizada en base a la norma INEN 1489 y normas ASTM.

Palabras Claves: biodiesel; higuerilla; propiedades fisicoquímicas

\begin{abstract}
This paper presents the physicochemical characterization of commercial diesel (Premium), and biodiesel based on castor oil, in proportion B10. A comparative analysis has been carried out regarding the characteristics obtained for both types of fuels and the results achieved are shown. The physicochemical properties treated are: Flash point, kinematic viscosity, basic sediments and water (BSW), corrosion to the copper sheet, sulfur and calculated cetane number. The properties of biodiesel are influenced by the physical characteristics of the fatty acid composition, such as the degree of unsaturation, the percentage of saturated fatty acids, monounsaturated fatty acids, and the polyunsaturated fatty acid. The properties of fuels are the key factors in determining the suitability of any alternative fuel. The results show that the B10 biodiesel characteristics are within the ranges stipulated by international regulations, in addition to significantly reducing polluting emissions. The characterization of fuels has been carried out based on the INEN 1489 standard and ASTM standards.
\end{abstract} Key words: castor oil biodiesel; physicochemical properties 


\section{Introducción}

La ASTM describe al biodiesel como una mezcla de mono-alquíl ésteres de triglicéridos y de ácidos grasos de cadena larga procedentes de aceites vegetales y grasas animales. Estos componentes se obtienen principalmente por la transesterificación con un alcohol de cadena corta (metanol, etanol o propanol), en presencia de un catalizador ácido, básico o enzimático (Barajas, 2004). (Benavides, 2004).

Un biodiesel óptimo se caracteriza por lograr que los motores no se expongan a mayores modificaciones, ni presenten problemas a mediano y largo plazo, además de que la potencia, torque y consumo no se vean afectados (Graboski \& McCormick, 1998).

Las características fisicoquímicas del biodiesel dependen de la materia prima de origen del aceite y del porcentaje de mezcla. Características tales como la densidad y viscosidad del combustible, tienen incidencia en el rendimiento del motor y sus emisiones. Sin embargo, de esto existen evidencias de que el deterioro del motor y su rendimiento, son muy similares al utilizar diésel fósil y biodiesel (A. Benavides, Benjumea, \& Pashova, 2007) (Pehan, Jerman, Kegl, \& Kegl, 2009).

La utilización de biodiesel presenta ventajas significativas, entre ellas se pueden mencionar: proviene de un recurso renovable, es biodegradable, reduce las partículas en más de un 50\% y las emisiones de $\mathrm{CO}_{2}$, está libre de benceno y aromatizantes altamente cancerígenos, incrementa la eficiencia y la durabilidad del motor, mejorando su ignición y lubricidad (Altin, Cetinkaya, \& Yucesu, 2001) (A. Benavides et al., 2007).

Las características de los ésteres son más parecidas a las del diésel fósil que las del aceite vegetal sin modificar. Además de esto se puede mencionar que la viscosidad de los ésteres es aproximadamente dos veces superior a la del biodiesel y el índice de cetano de los ésteres es superior, ratificando las ventajas de lubricidad en el sistema de inyección de motores CRD-i y calidad del combustible, esto debido al aumento del índice de cetano en el biodiesel (ASTM D6751-15c, 2010) (ASTM D6751, 2011).

En este trabajo se muestra la influencia que tiene el aceite de higuerilla en las propiedades de biodiesel B10 y sus características de inyección en sistemas CRD-i. Para esto se realiza un análisis comparativo entre las propiedades de biodiesel B10 y las de diésel de origen fósil. También se demuestra el potencial del biodiesel de higuerilla como una alternativa real al diésel de origen fósil en nuestro medio.

\section{Metodología}

La caracterización fisicoquímica del biodiesel B10 a base de higuerilla se ha realizado bajo métodos de ensayo de la "American Society for Testing and Materials" (ASTM), en los laboratorios de la Facultad de Ingeniería Química de la Universidad Central del Ecuador en Quito.

Para evaluar las propiedades del biodiesel se deberán cumplir los requisitos estipulados por la norma NTE INEN 2482 y en el caso del diésel fósil, los requisitos impuestos en la norma NTE INEN 1489. 


\section{Densidad}

Indica el contenido de energía en el combustible. Densidades mayores generan mayor energía térmica, buen rendimiento y economía del combustible (Ferella, Mazziotti Di Celso, De Michelis, Stanisci, \& Vegli, 2010). La densidad se la realizó bajo el método de ensayo PNE/DPEC/P/ASTM D-287.

\section{Punto de Inflamación}

Se establecen los productos volátiles para conocer la temperatura máxima a la que el fluido puede ser calentado sin ningún riesgo. Además, permite conocer si existe una cantidad excesiva de alcohol no reaccionado en el proceso de obtención del biodiesel (Ferella et al., 2010). El punto de inflamación se lo realizó bajo el método de ensayo PNE/DPEC/P/ASTM D92 PNE/DPEC/P/ASTM D-93.

\section{Sedimento básico y agua (BSW)}

El agua puede corroer componentes del motor, además de contribuir con el crecimiento de microorganismos creando lodos y saturando los filtros. Los sedimentos se forman debido a un mal proceso de purificación del combustible o contaminación del mismo, afectando principalmente a la temperatura de cristalización y al número de cetano (Benjumea P.N. Agudelo J. R., 2009) (Kaplan, Arslan, \& Sürmen, 2006). BSW se realizó bajo el método de ensayo ASTM D-4007.

\section{Viscosidad cinemática}

Una viscosidad mínima es de vital importancia para evitar mermas de potencia debido a fugas en la bomba de inyección y el inyector. Una viscosidad óptima generará un bombeado correcto en el sistema de inyección y proporcionará las características adecuadas de lubricidad (Pehan et al., 2009). Para obtener la viscosidad cinemática se utilizó el método de ensayo PNE/DPEC/P/ASTM D-445.

\section{Contenido de azufre}

El azufre coopera con el desgaste del motor y la formación de depósitos que varían dependiendo del funcionamiento de éste. Además, afecta al sistema de control de emisiones y límites medioambientales (Aardahl C., 2002). Para adquirir el contenido de azufre se empleó el método de ensayo PNE/DPEC/P/MI04 Ref. ASTM D-4294.

\section{Corrosión a la lámina de cobre}

La comprobación de la corrosión a la lámina de cobre permite observar si existen en el sistema compuestos corrosivos o presencia de ácidos que atacan al cobre o sus aleaciones (Karavalakis G. Stournas S., 2010). Se utilizó el método de ensayo PNE/DPEC/P/ASTM D-130.

\section{Número de cetano calculado}


Mesura de la calidad de ignición del combustible, influye en las emisiones de humo y en la calidad de la inflamación. Depende del diseño, cilindrada del motor, variaciones de carga, velocidad, condiciones de arranque y presión atmosférica. El bajo número de cetano induce ruidos en el motor, prolongando el retraso de la ignición y aumentando el peso molecular de las igniciones (Karavalakis G. Stournas S., 2010). Se utilizó el método de ensayo PNE/DPEC/P/ASTM D-287.

\section{Temperatura de destilación}

Muestra la máxima temperatura a la que se evaporará el combustible a condiciones de temperatura y presión dadas. La curva de destilación predice la composición del producto (Van Gerpen, 2004). Se utilizó el método de ensayo ASTM D-86.

\section{Residuo carbonoso sobre el $10 \%$ del residuo de destilación}

Indica la tendencia a formar depósitos de carbonilla en los elementos del motor (Van Gerpen, 2004). Se utilizó el método de ensayo ASTM D-189.

\section{Cenizas}

Las cenizas se componen de impurezas como arena y orín, que son tremendamente abrasivas, por lo tanto, el contenido de cenizas debe ser bajo para prevenir el desgaste en exceso de los elementos del motor (Kates E., 2003). Se utilizó el método de ensayo ASTM D-482.

\section{Resultados}

Para evaluar las características del biodiesel se deberán cumplir los requisitos estipulados por la norma NTE INEN 2482, mostrada en la tabla 1:

Tabla 1. Norma NTE INEN 2482: 2009-03

\begin{tabular}{|c|c|c|c|c|}
\hline Requisitos & Unidad & Mínimo & Máximo & $\begin{array}{l}\text { Método de } \\
\text { ensayo }\end{array}$ \\
\hline Densidad a $15^{\circ} \mathrm{C}$ & $\mathrm{Kg} / \mathrm{m}^{3}$ & 860 & 900 & ASTM D 1298 \\
\hline Punto de inflamación & ${ }^{\circ} \mathrm{C}$ & 120 & -- & ASTM D 93 \\
\hline Agua y sedimento & $\%$ & -- & 0,05 & ASTM D 1796 \\
\hline Viscosidad cinemática a $40^{\circ} \mathrm{C}$ & $\mathrm{mm}^{2} / \mathrm{s}$ & 3,5 & 5 & ASTM D 445 \\
\hline Cenizas sulfatadas & $\%(\mathrm{~m} / \mathrm{m})$ & -- & 0,02 & ASTM D 874 \\
\hline Contenido de azufre & $\mathrm{mg} / \mathrm{Kg}$ & -- & 10 & ASTM D 1552 \\
\hline Carbón residual & $\%$ & -- & 0,05 & ASTM D 4530 \\
\hline Corrosión lámina de cobre & Clasificación & -- & 3 & ASTM D 130 \\
\hline Número de cetano & - & 49 & -- & ASTM D 613 \\
\hline $\begin{array}{l}\text { Temperatura de destilación al } 90 \% \\
\text { recuperado }\end{array}$ & ${ }^{\circ} \mathrm{C}$ & -- & 360 & ASTM D 1160 \\
\hline
\end{tabular}

Así mismo para evaluar y comparar las propiedades fisicoquímicas con el diésel Premium de origen fósil se emplea la norma NTE INEN 1489, mostrada en la tabla 2: 
Tabla 2. Norma NTE INEN 1489 Octava revisión

\begin{tabular}{|c|c|c|c|c|}
\hline Requisitos & Unidad & Mínimo & Máximo & $\begin{array}{l}\text { Método de } \\
\text { ensayo }\end{array}$ \\
\hline Punto de inflamación & ${ }^{\circ} \mathrm{C}$ & 51 & -- & ASTM D 93 \\
\hline Agua y sedimento & $\%$ & -- & 0,05 & ASTM D 2709 \\
\hline Viscosidad cinemática a $40^{\circ} \mathrm{C}$ & $\mathrm{mm}^{2} / \mathrm{s}$ & 2 & 5 & ASTM D 445 \\
\hline Cenizas sulfatadas (fracción de masa) & $\%$ & -- & 0,01 & ASTM D 482 \\
\hline Contenido de azufre (fracción de masa) & $\%$ & -- & 0,05 & $\begin{array}{l}\text { ASTM D } 2622 \\
\text { ASTM D } 4294 \\
\text { ASTM D } 5453\end{array}$ \\
\hline $\begin{array}{l}\text { Contenido de residuo carbonoso (fracción } \\
\text { de masa) }\end{array}$ & $\%$ & -- & 0,1 & ASTM D 4530 \\
\hline Corrosión lámina de cobre & Clasificación & -- & $1^{\mathrm{a}}$ & ASTM D 130 \\
\hline Índice de cetano calculado & - & 45 & -- & ASTM D 613 \\
\hline $\begin{array}{l}\text { Temperatura de destilación al } 90 \% \\
\text { recuperado }\end{array}$ & ${ }^{\circ} \mathrm{C}$ & -- & 360 & ASTM D 86 \\
\hline $\begin{array}{l}\text { Contenido de biodiesel (fracción de } \\
\text { volumen) }\end{array}$ & $\%$ & 5 & 10 & EN 14078 \\
\hline
\end{tabular}

Los datos obtenidos del análisis fisicoquímico del biodiesel a base de aceite de higuerilla mezclan B10 realizado en el Departamento de Petróleos, Energía y Contaminación, de la Facultad de Ingeniería Química de la Universidad Central del Ecuador están expuestos en la tabla 3:

Tabla 3. Análisis fisicoquímico del biodiesel a base de aceite de higuerilla B10 (Condiciones Ambientales: Presión 542,9 mm Hg; Temperatura: $17,5^{\circ} \mathrm{C}$ )

\begin{tabular}{|c|c|c|c|}
\hline Determinación & Unidad & Método de ensayo & Resultado \\
\hline Densidad API a $60{ }^{\circ} \mathrm{F}$ & ${ }^{\circ} \mathrm{API}$ & PNE/DPEC/P/ASTM D.287 & 34,0 \\
\hline Punto de inflamación & ${ }^{\circ} \mathrm{C}$ & $\begin{array}{l}\text { PNE/DPEC/P/ASTM } 0.92 \\
\text { PNE/DPEC/P/ASTM } 0.93\end{array}$ & 72,1 \\
\hline Agua y sedimento & $\% \mathrm{~V}$ & ASTM D-4007 & 0,05 \\
\hline Viscosidad cinemática a $40^{\circ} \mathrm{C}$ & $\mathrm{mm}^{2} / \mathrm{s}$ & PNE/DPEC/P/ASTM D-445 & 4,21 \\
\hline Cenizas sulfatadas & $\% \mathrm{P}$ & ASTM D.482 & 0,0080 \\
\hline Contenido de azufre & $\% \mathrm{P}$ & $\begin{array}{l}\text { PNE/DPEC/P/MI04 Ref. ASTM D- } \\
4294\end{array}$ & 0,0148 \\
\hline $\begin{array}{l}\text { Contenido de residuo } \\
\text { carbonoso }\end{array}$ & $\% \mathrm{P}$ & ASTM D· 189 & 0,13 \\
\hline Corrosión lámina de cobre & Clasificación & PNE/DPEC/P/ASTM D· 130 & $1 \mathrm{~b}$ \\
\hline Índice de cetano calculado & - & PNE/DPEC/P/ASTM D-287 & 51 \\
\hline $\begin{array}{l}\text { Temperatura de destilación al } \\
90 \% \text { recuperado }\end{array}$ & ${ }^{\circ} \mathrm{C}$ & ASTM D 86 & 350,7 \\
\hline
\end{tabular}

Los valores de densidad fuera de los rangos establecidos por las normativas ocasionarían problemas de combustión que pueden ser por retrasos en la inyección con el consiguiente deterioro de los elementos del motor (Torres J.E. Jerman M.S., 2011). El resultado obtenido es de $855 \mathrm{Kg} / \mathrm{m}^{3}$, el cual es ligeramente mayor al del diésel de origen fósil $850 \mathrm{Kg} / \mathrm{m}^{3}$, y se encuentra dentro de los rangos expuestos en la tabla 1.

El punto de inflamación en el biodiesel es mayor al del diésel fósil $72,1^{\circ} \mathrm{C}$ frente a un promedio de $52{ }^{\circ} \mathrm{C}$ respectivamente, lo cual es bastante beneficioso, ya que se puede emplear en motores con mayores tasas de compresión, los mismos que erogan más potencia y torque. 
El contenido de agua, aunque se encuentra en el límite, cumple con los rangos establecidos en la tabla 1 , que es de $0,05 \%$ en volumen, al igual que el diésel fósil; una excesiva concentración de agua provocaría la separación de fases en las mezclas con diésel fósil, así como fomentar la corrosión y presencia de microorganismos que degraden el biodiesel.

La viscosidad medida es superior $4,21 \mathrm{~mm}^{2} / \mathrm{s}$ frente a un promedio de $2,5 \mathrm{~mm}^{2} / \mathrm{s} \mathrm{del}$ diésel de origen fósil, con el consiguiente requerimiento de un sistema de inyección de mayor presión para evitar problemas en la combustión, debido a la variación del flujo del combustible (Alptekin \& Canakci, 2009). Los sistemas de inyección CRD-i caben perfectamente con lo mencionado debido a que estos trabajan con presiones bastante elevadas.

Las cenizas sulfatadas $0,0080 \%$ en peso, cumplen con el requerimiento de la norma $0,02 \%$, por lo que no generarán depósitos adicionales. El contenido de residuo carbonoso excede el límite de la norma en 0,08\%, esto debido al alto porcentaje en peso del residuo de carbón en el aceite de ricino 0,22\% (Morales F.; Vázquez, 2012) (Pérez M.; Perdomo, 2012).

Finalmente, las pruebas que al biodiesel de higuerilla B10 lo catalogan como combustible son el número de cetano, el cual es afectado por la cantidad de metanol empleado en el proceso de la transesterificación, éste es superior al del diésel fósil, 51 de acuerdo con el método de ensayo ASTM D- 287, mostrada en la tabla 3. Mejorando notoriamente el proceso de combustión, sin afectar la entrega de potencia y par motor (Randazzo \& Sodré, 2011).

Así mismo la reducción del contenido de azufre en el combustible contribuye con la vida útil del motor, ya que cuando el diésel fósil con un mayor contenido de azufre se consume en la cámara de combustión, se forman óxidos de azufre que reaccionan con el vapor de agua para formar el ácido sulfúrico. Al igual que el sulfuro de hidrógeno, si estos vapores de ácidos se condensan, atacan químicamente las superficies de metal de las guías de válvula, de las camisas de los cilindros y pueden afectar los cojinetes (Randazzo \& Sodré, 2011).

La corrosión en la lámina de cobre de acuerdo al método de ensayo ASTM D·130, arrojó una clasificación $1 b$ "ligero empañamiento" lo que indica que el biodiesel de higuerilla B10 no provoca corrosión en ningún elemento del sistema de combustible, además corrobora en que el biodiesel puede estar almacenado por periodos de tiempo relativamente largos sin modificaciones mayores en su composición (Karavalakis G. Stournas S., 2010) (Randazzo \& Sodré, 2011).

\section{Conclusiones}

La calidad del biodiesel a base de aceite de higuerilla B10 difiere del biodiesel de origen fósil, lo cual se debe al proceso de obtención de cada uno, el primero es generado artesanalmente en un laboratorio y el segundo proviene de un proceso de refinación a nivel industrial. Lo antes mencionado, es evidenciado en los resultados de la caracterización, al cumplir o no con los valores estipulados en las Normas NTE INEN 2482, NTE INEN 1489. 
La caracterización de ciertas propiedades fisicoquímicas del biodiesel, permiten conocer la calidad de este, al cumplir o no con los límites permisibles estipulados en las correspondientes normas.

Si se aumenta la cantidad de metanol en el proceso de transesterificación para la obtención del biodiesel B10 mejorará el número de cetano y el contenido de éster.

El valor de la viscosidad cinemática del biodiesel B10 casi dobla el valor del diésel fósil, lo que coadyuva en la utilización de este biocombustible en sistema de inyección con mayores presiones de trabajo como son los CRD-i, con presiones que exceden los 2000 bares.

La utilización del biodiesel B10 corrobora con las normativas medioambientales, debido a que se reduce del $0,05 \%$ contenido en el diésel de origen fósil a $0,0184 \%$ en peso presente en el biodiesel de higuerilla B10, esto debido a la reducción en las emisiones de $\mathrm{SO}_{\mathrm{x}}$ de los gases producidos en la combustión.

El resultado arrojado en la tabla 3 respecto a la corrosión en la lámina de cobre, indica que el biodiesel B10 se encuentra en la clasificación 1b, lo que se traduce en la familiaridad de éste con las piezas o elementos del motor, sin modificación alguna de estos elementos, sobretodo en el sistema de combustible.

Se instituyen puntos de partida para nuevos proyectos como, conocer los efectos luego de las correcciones en la reacción de transesterificación; efectos al ser utilizado en mezclas como B5, B15 en motores Diésel con sistemas de inyección CRD-i, cuantificación de las emisiones, desempeño y los efectos a corto y largo plazo.

\section{Bibliografía}

Aardahl C. (2002). Plasma-activated lean NOx catalysis for heavy-duty diesel emissions control. 8th Diesel Engine Emissions Reduction Conference, San Diego, Calif.

Alptekin, E., \& Canakci, M. (2009). Characterization of the key fuel properties of methyl ester-diesel fuel blends. Fuel, 88(1), 75-80. https://doi.org/10.1016/j.fuel.2008.05.023

Altin, R., Cetinkaya, S., \& Yucesu, H. (2001). Potential of Using Vegetable Oil Fuels as Fuel for Diesel Engines. Energy Conversion and Management, 42, 529-538.

ASTM D6751. (n.d.). Standard Specification for Diesel Fuel Oils. ASTM, West Conshohocken, PA., (25).

ASTM D6751-15c. (2010). Standard Specification for Biodiesel Fuel Blend Stock (B100) for Middle Distillate Fuels. ASTM International, $i, 1-11$. https://doi.org/10.1520/D6751

Barajas, C. L. (2004). Obtención de biodiésel a partir de la higuerilla (ricinus communis). ler. Seminario Internacional de Biocombustibles. Bogotá: Universidad Nacional de Colombia Sede Bogotá. 
Benavides, A., Benjumea, P., \& Pashova, V. (2007). El biodiesel de aceite de higuerilla como combustible alternativo para motores diesel. Dyna, 74(153), 141-150. Retrieved from https://revistas.unal.edu.co/index.php/dyna/article/view/951

Benavides, A. Y. (2004). Biodiésel de aceite de higuerilla. Una evaluación de su obtención y utilización. Trabajo dirigido de grado. Universidad Nacional de Colombia Sede Medellín.

Benjumea P.N. Agudelo J. R., R. L. A. (2009). Biodiesel: Producción, Calidad y Caracterización. 1ra Edición. Editorial Universidad de Antioquia. Medellín.

Ferella, F., Mazziotti Di Celso, G., De Michelis, I., Stanisci, V., \& Vegli, F. (2010). Optimization of the transesterification reaction in biodiesel production. Fuel, 89(1), 36-42. https://doi.org/10.1016/j.fuel.2009.01.025

Graboski, M. S., \& McCormick, R. L. (1998). Combustion of fat and vegetable oil derived fuels in diesel engines. Progress in Energy and Combustion Science, 24(2), 125-164. https://doi.org/10.1016/S0360-1285(97)00034-8

Kaplan, C., Arslan, R., \& Sürmen, A. (2006). Performance characteristics of sunflower methyl esters as biodiesel. Energy Sources, Part A: Recovery, Utilization and Environmental Effects, 28(8), 751-755. https://doi.org/10.1080/009083190523415

Karavalakis G. Stournas S., K. D. (2010). Evaluation of the oxidation stability of diesel/biodiesel blends. Elsevier, Fuel, (89), 2483-2489.

Kates E., L. W. (2003). Motores Diésel y de gas de alta compresión. Editorial Reverté, S.A., 192.

Morales F.; Vázquez, J. C. . H. J. . S. P. (2012). Evaluación del aceite de higuerilla del istmo de Tehuantepec para la producción de biodiésel. Revista Latinoamericana de Química. Laboratorios Mixim, S.A. de C.V. México., 146.

Pehan, S., Jerman, M. S., Kegl, M., \& Kegl, B. (2009). Biodiesel influence on tribology characteristics of a diesel engine. Fuel, 88(6), 970-979. https://doi.org/10.1016/j.fuel.2008.11.027

Pérez M.; Perdomo, F. . M. B. . R. M. (2012). Diseño, síntesis y caracterización de un biodiésel combustible $(2 \mathrm{G})$ energéticamente óptimo a partir de mezclas de aceite de higuerilla y grasas ácidas. Centro de Física Aplicada Y Tecnología Avanzada. Universidad Nacional Autónoma de México. Querétaro-México.

Randazzo, M. L., \& Sodré, J. R. (2011). Exhaust emissions from a diesel powered vehicle fuelled by soybean biodiesel blends (B3-B20) with ethanol as an additive (B20E2-B20E5). Fuel, 90(1), 98-103. https://doi.org/10.1016/j.fuel.2010.09.010

Torres J.E. Jerman M.S., G. A. L. I. D. M. P. K. B. (2011). Physical and chemical properties of ethanoldiesel fuel blends. Elsevier, Fuel, 90, 795-802.

Van Gerpen, J. (2004). Biodiesel production technology. National Renewable Energy Laboratory, 1617. 
